\title{
Los retos del periodismo ecuatoriano y los desafíos en la formación de nuevos profesionales
}

\section{The challenges of Ecuadorian journalism and the challenges in the training of new professionals}

Lcda. Julissa Villanueva Barahona, MAE

Lcda. Karla López Cedeño, MsC

Lcdo. José Omar Villavicencio, MsC

Universidad Laica Vicente Rocafuerte de Guayaquil, Ecuador

Lcda. Dennys Jordán Correa, MsC

Universidad Internacional del Ecuador, Ecuador

Autor para correspondencia: jvillanuevab@ulvr.edu.ec; kclopezc@ulvr.edu.ec;

jvillavicencios@ulvr.edu.ec; dejordanco@uide.edu.ec

Fecha de recepción: 10 de junio de 2018 - Fecha de aceptación: 15 de septiembre de 2018

Resumen: Al forjar profesionales de la comunicación es preciso y muy necesario bosquejar una imagen real del perfil del periodista frente a los retos del periodismo ecuatoriano y de las nuevas tendencias comunicacionales. Uno de ellos es la especialización; es decir la exigencia de peritos multifacéticos que dominen un espectro amplio de conocimiento y no solo en determinadas áreas, según los requerimientos mediáticos e informativos. Se promueve una evolución y crecimiento de las tendencias informativas digitales frente a las tradicionales lo que implica un crecimiento de emprendimientos bajo el paraguas de periodismo independiente por lo que surge un nuevo tipo de periodismo especializado en el mundo digital o multiplataforma. Sin embargo, esta especialización exige la revisión de competencias que se forjan en la academia y en la manera que se imparten como una forma de forjar el futuro integral de los estudiantes.

Palabras Claves: periodismo ecuatoriano; desafíos; formación especializada; formación académica

\begin{abstract}
When forging communication professionals it is necessary and very necessary to write a real image of the profile of the period facing the challenges of Ecuadorian journalism and new communication trends. One of them is specialization; that is, the demand of multifaceted experts who dominate a broad spectrum of knowledge and not only in certain areas, according to media and information requirements. It promotes an evolution and growth of digital informational tendencies compared to the traditional ones that imply a growth of ventures under the umbrella of independent journalism, which is why a new kind of specialized journalism arises in the digital or multiplatform world. However, this specialization requires the revision of the competences that are taught in the academy and in which they are taught as a way of the future.
\end{abstract}

Key words: ecuadorian journalism; challenges; specialized training 


\section{Introducción}

En 75 años la formación de periodistas en Ecuador ha evolucionado a un ritmo vertiginoso; desde la creación de la primera Facultad de Comunicación Social de la Universidad Central del Ecuador (Quito) en 1943 hasta el éxodo del ejercicio profesional a las plataformas digitales en este nuevo milenio. El perfil del periodista, demanda cada vez más de profesionales con mayores competencias y que tenga la capacidad de asumir en algún momento el rol de autor/editor que publica su propio material. Eso implica una preparación que va más allá de entender -de forma intuitiva- las herramientas web y la inmediatez que demanda el medio (Calvaresi, Valdivia, Foropón, \& Luna, 2010).

El campo laboral para periodistas en Ecuador se concentra en medios de comunicación privados, aunque en los últimos años se han registrado esfuerzos por democratizar su área de acción. Según publicación del Sistema de Información Oficial El Ciudadano, en el 2006 existían 1.404 medios de comunicación privados, 117 públicos y ni un solo medio comunitario; pero al 2014 el registro varió a 1.324 medios privados, 344 públicos y 20 medios comunitarios (El Ciudadano, 2014).

La información especializada colgada en portales web de comunidades de periodistas identifican estudios relacionados sobre el periodismo especializado y las tendencias en esta rama de las ciencias sociales, debido a que cada vez es más cambiante el modo de acceder a información y la disponibilidad de herramientas para su procesamiento. A través del método lógico deductivo se trazó la ruta para interrelacionar conceptos, casos, marco legal y teórico para profundizar en el problema identificado. Para su desarrollo se aplicó la técnica de la entrevista a representantes del periodismo local, a quienes se les consultó cuáles son los principales requerimientos del perfil profesional actual; así como fortalezas y debilidades de los neoperiodistas que son enrolados en los medios de comunicación y evidenciar las necesidades que podrían ser cubiertas desde las aulas de clases.

Como referente de la presente investigación se consultó a periodistas especializados en diferentes áreas de la comunicación y que ocupan cargos jerárquicos; todos con más de 15 años de experiencia. Además se contrastó la información con profesionales internacionales, quienes paralelamente ejercen la docencia; así se definieron lineamientos importantes sobre los requerimientos del perfil periodístico, las fortalezas y debilidades del profesional recién egresado de las universidades comparado con aquellos que poseen experiencia, según se observa en la tabla 2 .

\section{Procesos y construcción del periodismo nacional como ejercicio y proceso formativo del país}

Antes de la promulgación de la Ley Orgánica de Comunicación, aprobada el 25 de junio del 2013, el ejercicio periodístico carecía de un marco legal que regulara su contratación en medios de comunicación social; pero, este aspecto cambió desde su vigencia, según se puntualiza en el artículo en la Sección II, Derechos de la Comunicación.

Art. 42: Las actividades periodísticas de carácter permanente realizadas en los medios de comunicación, en cualquier nivel o cargo, deberán ser desempeñadas por profesionales en periodismo o comunicación, con excepción de las personas que tienen espacios de opinión, y 
profesionales o expertos de otras ramas que mantienen programas o columnas especializadas (...) En las entidades públicas los cargos inherentes a la comunicación serán desempeñados por comunicadores o periodistas profesionales.

A partir de este punto la atención para el cumplimiento de este artículo se centró en dos ejes:

- Lograr la profesionalización de periodistas y comunicadores en general.

- Reforzar la preparación académica en las universidades, que atraviesan un proceso de categorización.

\section{La profesionalización de los periodistas}

En el primer eje se destaca la Ley de Ejercicio Profesional en Ecuador promulgada en el gobierno de Guillermo Rodríguez Lara (1972-1976), que permitió la certificación de periodistas por los años de ejercicio profesional antes que por acudir a la universidad; mientras que durante el régimen de Rafael Correa Delgado (2007-2017) se promovió la profesionalización de este grupo de comunicadores sin título superior a través de capacitaciones de actualización, por parte del Estado.

Tabla 1. Principales hechos que incidieron en el Periodismo de Ecuador

\begin{tabular}{|c|c|}
\hline \multirow[b]{2}{*}{ Año } & Efeméride Periodismo en Ecuador \\
\hline & Descripción \\
\hline 1943 & Creación de la primera Facultad de Comunicación Social, Universidad Central del Ecuador (Quito) \\
\hline 1945 & Creación Escuela de Información, Universidad de Guayaquil (Guayaquil) \\
\hline 1969 & Surge Internet a nivel mundial \\
\hline 1975 & $\begin{array}{l}\text { Promulgación Ley de Ejercicio Profesional en Ecuador (cualquier ciudadano que avale } 5 \text { años de } \\
\text { experiencia podía acceder a un título profesional) }\end{array}$ \\
\hline 2013 & Vigencia de La Ley Orgánica de Comunicación (LOC), Ecuador \\
\hline & Constitución Superintendencia de Comunicación (Supercom), Ecuador \\
\hline & $\begin{array}{l}\text { Constitución Consejo de Regulación y Desarrollo de la Información y Comunicación (Cordicom), } \\
\text { Ecuador }\end{array}$ \\
\hline
\end{tabular}

Fuentes: Cordicom, Repositorio Universidad Central del Ecuador

Un dato importante a considerar dentro del contexto periodístico es que en el país hay 17.000 trabajadores de los medios de comunicación, de los cuales un $36 \%$ corresponde a mujeres, según el Registro Público de Medios (Cordicom, 2015).

Esta cifra, sumada al número de egresados anual de las universidades, enciende las alertas sobre la dificultad de colocar a los jóvenes profesionales en el ámbito laboral; y, da paso a un mercado del contexto interdisciplinario de la comunicación como lo es el emprendimiento de productos periodísticos que ha ganado espacio en la última década; esto como una forma de descongestionar la oferta de periodistas ante una limitada demanda de profesionales.

Estas propuestas dan muestra significativa de la evolución y crecimiento de las tendencias informativas digitales frente a las tradicionales. Unos ejercen estos emprendimientos bajo el paraguas de periodismo independiente, entendiéndose así a aquellos medios nativos en la web; y, a otros, correspondientes a los medios tradicionales que se vieron obligados a ampliar su presencia con las audiencias online. Surge así, un nuevo tipo de periodismo especializado en el mundo digital o multiplataforma. 


\section{El periodismo Data dentro del quehacer web}

En las salas de redacción de los medios tradicionales, los requerimientos también están enfocados al manejo de recursos digitales y un perfil del periodista multiplataforma, que integre los ya posicionados ecosistemas del Periodismo Data o de Datos (PD).

Schudson (2010) relacionó el uso de bases de datos en la producción de noticias con prácticas de recolección y estructuración de datos que los observatorios políticos pusieron en marcha a partir de la década de los 70. Manovich (2001) ya aseguraba que las bases de datos son para la era digital lo que la narrativa fue para la edad moderna de la novela y el cine. Este futuro de las noticias en 2010 representa el hoy de las redacciones con equipos de PD, en donde las bases de datos liberadas por grandes filtraciones - como es el caso de los Papeles de Panamá o la Lista Falciani- o a través de portales de transparencia gubernamental, es una de las características principales del ejercicio periodístico en el PD (La-Rosa \& Sandoval-Martín, 2016, pág. 1211).

Se vive, entonces, una evolución exponencial del PD tras la llegada de internet, tal como detalla Ávila (2013):

Entre 2005 y 2007 comenzaron a surgir herramientas digitales y tecnologías a precios accesibles para el manejo de datos. También en 2007 la Knight Foundation premió el proyecto Everyblock que permitía a los usuarios conocer información sobre su barrio gracias a una programación derivada de una base de datos (Ferrerez, 2012: 121). En 2009 el St. Petersburg Times recibió el Premio Pulitzer por el proyecto Politifact. En ese año el jurado calificador dijo que el trabajo "demostraba que los periodistas junto con el poder de internet habían podido separar los hechos de la retórica de las campañas electorales para iluminar a los votantes". Jack McElroy, editor del Knoxville News Sentinel, dijo "las bases de datos en línea se están convirtiendo rápidamente en una herramienta importante para el periodismo watchdog en la era digital. Al identificar a Politifact como el mejor reporteo del año, se va a acelerar esta tendencia".

En consecuencia, uno de los desafíos del periodista actual es saber cómo procesar ese mar de datos que ya está disponible en el ciberespacio, sobre todo a nivel de Gobiernos pero que requieren ser encontrados, procesados y explicados. Para ello se destaca que los "ocho principios del Open Government Data hablan de la necesidad de datos públicos, completos, originales, fechados/datados, accesibles, electrónicos, sin discriminar el acceso a cualquier persona" (Antón Bravo, 2013, pág. 108).

Ante este escenario surge el reto para que los periodistas en formación salgan de las aulas con la capacidad adicional de cubrir un mercado que demanda profesionales con dominio en las técnicas de procesamiento de datos en temas políticos, económicos, científicos, de salud, comunitarios, judiciales, entre otras especialidades; así como atender los espacios del periodismo digital, a los que ya se han integrado los medios tradicionales y que, para los medios nativos digitales, también representa una exigencia. 
La Fundación Andina para la Observación y Estudio de Medios (Fundamedios) trazó un mapa sobre los medios nativos digitales en Ecuador. Hasta abril de 2015, este mapa está integrado por 60 organizaciones o medios, conforme se observa en la tabla 3 (Anexos) y en donde se destaca el desarrollo de propuestas de periodismo especializado.

Los temas de especialidad periodística que requieren mayor atención entre los medios, por su sensibilidad e interés con las audiencias, son aquellos relacionados con Economía, Política, Judiciales o Sucesos Policiales, Interculturalidad y Periodismo Deportivo. En el libro 'Presente y futuro en el Periodismo Especializado' se marcan las pautas para atender estas grandes áreas de la información, en cuanto a métodos de investigación, redacción, formas y criterios periodísticos.

Por ejemplo, Maritza Sobrados trata en profundidad la información económica. Para ello recopila los principios del buen hacer en esta materia:

Rigurosidad, buena divulgación, profundización de los acontecimientos (...) detalla la polémica relación del periodista económico con sus fuentes y los retos en el manejo del contexto informativo, como reflexión sobre el papel de los medios en la crisis económica. García Gordillo aborda la comunicación política, describiendo la tematización en la que ésta especialidad se desglosa (información local, nacional, comunitaria e internacional); las características de las fuentes y la imbricación del lenguaje político. Mientras que para la profesora de la Universidad Complutense Concha Fagoaga, el periodismo especializado, especialmente en los soportes digitales, se identifica con el futuro del periodismo. Pero el futuro es siempre, y especialmente en estos tiempos, una incertidumbre.

Hay otros temas de especialidad que empezaron a marcar su ruta de desarrollo; sin embargo, no muestran la atención requerida en el campo periodístico. Por ejemplo, aquellos relacionados con la disciplina científica, como la "información espacial", que surge como un nuevo campo laboral ante las constantes misiones fuera del planeta Tierra y las perspectivas por abrir un mercado relacionado al turismo espacial (Nadjejda, 2009, págs. 343, 344):

El periodista espacial estará a caballo entre la especialización científica y la popularización de los asuntos cósmicos. (...) Este tipo de periodista "todoterreno" se convierte en una apetecible figura para el análisis dentro de las teorías de la comunicación. (...) La repercusión mediática de los vuelos orbitales para civiles adinerados promete convertirse en información atractiva...

La era digital modificó el espacio laboral y las competencias profesionales porque incorporar la web en las jornadas periodísticas ha conllevado la organización de la producción, una convergencia de lenguaje y forjar entornos más colaborativos (Scolari, Micó, Navarro, \& Pardo, 2008).Se busca un perfil polifuncional, capaz de administrar información, generar la propia, diagramarla en formatos atractivos, difundirla de manera inmediata y programarla en todos los servidores; tal como menciona Scolari, se trata de un periodista que incorpore habilidades de diseñador y programador. 
Este panorama se coteja en Ecuador. Es imperativo forjar periodistas especializados y este tema es recurrente en foros de comunicación, portales informativos, blogs y grupos para capacitaciones que mencionan la necesidad de un profesional idóneo, término que implica el conocimiento de aspectos básicos como redacción, capacidad de respuesta inmediata y de generar información de manera inmediata y difundirla de diferentes plataforma; un profesional ético que aplique las normas de la profesión y demuestre predisposición para asumir que la profesión requiere presión. Así sintetizan los directores de medios de comunicación consultados.

Tabla 2. Necesidad de periodistas especializados en las salas de redacción

\begin{tabular}{|c|c|c|c|c|}
\hline Preguntas & $\begin{array}{c}\text { Lcda. Mónica } \\
\text { Mendoza, } \\
\text { Macroeditora } \\
\text { Grupo El Comercio }\end{array}$ & $\begin{array}{c}\text { Lcda. Marjorie } \\
\text { Ortiz, } \\
\text { Editora de } \\
\text { Investigación } \\
\text { Diario El } \\
\text { Universo }\end{array}$ & $\begin{array}{c}\text { Lcdo. Johnny } \\
\text { Alvarado, Editor } 1 \\
\text { Diario El Telégrafo }\end{array}$ & $\begin{array}{c}\text { Lcdo. José Toledo, } \\
\text { Director de la } \\
\text { plataforma de } \\
\text { noticias RTW24 }\end{array}$ \\
\hline $\begin{array}{l}\text { ¿Existe la } \\
\text { necesidad de } \\
\text { contratar } \\
\text { periodistas } \\
\text { especializados } \\
\text { en las salas de } \\
\text { redacción? }\end{array}$ & $\begin{array}{l}\text { El periodismo } \\
\text { mantiene sus } \\
\text { principios de informar } \\
\text { de forma oportuna, } \\
\text { veraz, con la mayor } \\
\text { cantidad de fuentes, } \\
\text { revisión de datos y su } \\
\text { contrastación de } \\
\text { forma responsable. } \\
\text { Con ayuda de la } \\
\text { tecnología, los } \\
\text { parámetros } \\
\text { búsqueda de } \\
\text { presentación de la } \\
\text { información son más } \\
\text { exigentes. En las salas } \\
\text { de redacción se busca } \\
\text { a profesionales que } \\
\text { dominen } \\
\text { periodismo data, las } \\
\text { herramientas digitales } \\
\text { que permitan } \\
\text { identificar, } \\
\text { ejemplo, si lo que dice } \\
\text { una autoridad está } \\
\text { acorde a la realidad, si } \\
\text { hay un antecedente y } \\
\text { para eso está el fact } \\
\text { checking vor } \\
\text { verificación } \\
\text { discurso (...) del }\end{array}$ & $\begin{array}{l}\text { El periodismo más } \\
\text { necesario en las } \\
\text { redacciones es el } \\
\text { periodismo de } \\
\text { investigación, } \\
\text { porque al tener } \\
\text { desarrolladas las } \\
\text { habilidades y } \\
\text { herramientas en } \\
\text { esta área puedes } \\
\text { especializarte en } \\
\text { cualquier sección. }\end{array}$ & $\begin{array}{l}\text { Es necesaria la } \\
\text { especialización, } \\
\text { incluso como forma de } \\
\text { diversificar la } \\
\text { profesión. } \\
\text { periodista } \\
\text { especializado da más } \\
\text { credibilidad al medio; } \\
\text { el lector sentirá } \\
\text { confianza al leer sus } \\
\text { notas porque, ampliará } \\
\text { conceptos, aportará } \\
\text { mayor contexto y } \\
\text { dominará el tema; esto } \\
\text { marcará la diferencia } \\
\text { con un periodista } \\
\text { normal que debe } \\
\text { afrontar el hecho de ser } \\
\text { "todólogo" cuando en } \\
\text { realidad no lo es. }\end{array}$ & $\begin{array}{l}\text { Un periodista } \\
\text { especializado tiene } \\
\text { una visión macro de } \\
\text { los temas de interés. } \\
\text { Está capacitado para } \\
\text { cubrir un evento o } \\
\text { noticia con el } \\
\text { lenguaje } \\
\text { especializado que lo } \\
\text { caracteriza. } \\
\text { permite a los medios } \\
\text { presentar noticias } \\
\text { exclusivas, al tener a } \\
\text { su disposición una } \\
\text { amplia agenda de } \\
\text { contactos en un tema } \\
\text { en específico y el } \\
\text { conocimiento para } \\
\text { escoger un enfoque o } \\
\text { eje que marque la } \\
\text { diferencia respecto a } \\
\text { las notas que } \\
\text { publique a } \\
\text { competencia. }\end{array}$ \\
\hline
\end{tabular}

De esa El periodista debe especialización, ¿qué tendencias requieren en su medio de comunicación? conocer de leyes, de economía. Con el Periodismo Data se logra profundidad de la información; por
Lo que más se $\mathrm{Se}$ demanda es el especialistas en periodismo de crónicas urbanas y registro... Se temas de salud. Todo requiere de periódico debe contar periodistas con ellos; pues atrás
Las áreas con mayor demanda son política, economía y seguridad (crónica). Sobre éstas debe existir un periodista 


$\begin{array}{llllr}\text { eso, en El Comercio } & \text { multimedia, que quedaron } & \text { los } & \text { multimedia } & \text { que } \\ \text { existe un área } & \text { van a una } & \text { redactores que rotaban } & \text { pueda } & \text { usar } \\ \text { especializada. } & \text { cobertura y bien } & \text { de sección en sección. } & \text { correctamente las } \\ & \text { termina } & \text { herramientas } & \\ & \text { autoridad } & \text { la } & \text { tecnológicas y de } \\ & \text { entrevistado de } & \text { web para informar } \\ & \text { hablar, ya tienen } & \text { con inmediatez y } \\ & \text { que enviar la } & \text { veracidad. } & \\ & \text { información tanto } & \\ & \text { en video, audio, } & \end{array}$

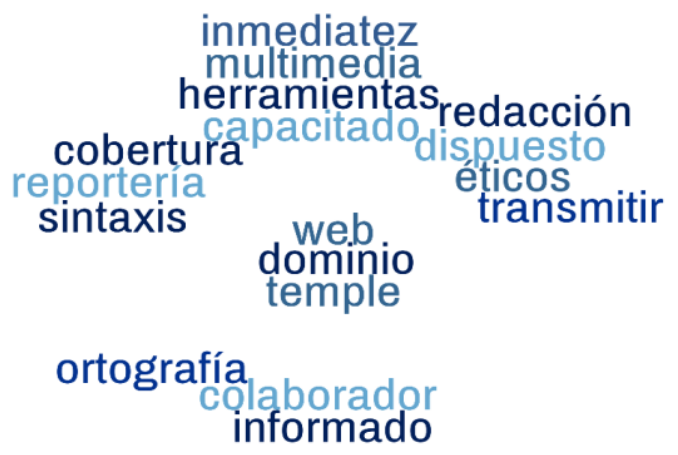

Figura 1. Terminología requerida para forjar un perfil periodístico idóneo.

Así como el profesional se va especializando en determinadas áreas, las audiencias viven similar transformación y los niveles de exigencia de información son mayores. Ir a ese ritmo de adaptaciones y cambios también representa un beneficio para que el periodista que encuentra oportunidades en el campo laboral o crean otras, con el soporte de las plataformas digitales.

El periodismo mantiene sus principios de informar de forma oportuna, veraz, con la mayor cantidad de fuentes, revisión de datos y su contrastación de forma responsable. Ahora, con ayuda de la tecnología, los parámetros de búsqueda y presentación de la información son más exigentes. "En las salas de redacción se busca a profesionales que dominen el periodismo data", enfatiza Mendoza argumentando que el manejo de herramientas digitales deberá enfocarse en identificar la veracidad de un discurso y la consecuente delimitación de antecedentes y fuentes porque es preciso mejorar la búsqueda de información y profundidad de la misma.

La especialización de un periodista marca una visión macro de los temas de interés. Garantiza el manejo de un lenguaje especializado que lo caracteriza y lo convierte en presentador de noticias exclusivas, así determina José Toledo, director de la plataforma de noticias RTW24. "Posee, también una amplia agenda de contactos en un tema y un conocimiento suficiente para buscar un enfoque o eje que marque la diferencia".

Tabla 3. Fortalezas y debilidades de los egresados que hacen pasantías en su medio de comunicación 


\begin{tabular}{|c|c|c|c|c|}
\hline Preguntas & $\begin{array}{c}\text { Lcda. Mónica } \\
\text { Mendoza, } \\
\text { Macroeditora } \\
\text { Grupo El Comercio }\end{array}$ & $\begin{array}{c}\text { Lcda. Marjorie } \\
\text { Ortiz, } \\
\text { Editora de } \\
\text { Investigación } \\
\text { Diario El } \\
\text { Universo }\end{array}$ & $\begin{array}{c}\text { Lcdo. Johnny } \\
\text { Alvarado, Editor } \\
\mathbf{1} \\
\text { Diario El } \\
\text { Telégrafo } \\
\end{array}$ & $\begin{array}{l}\text { Lcdo. José Toledo, } \\
\text { Director de la } \\
\text { plataforma de } \\
\text { noticias RTW24 }\end{array}$ \\
\hline $\begin{array}{lr}\text { ¿Cuáles son las } \\
\text { fortalezas } & \text { que } \\
\text { evidencian } & \text { los } \\
\text { pasantes } & \text { que } \\
\text { asisten a } & \text { su } \\
\text { medio } & \text { de } \\
\text { comunicación o } \\
\text { los contratados } \\
\text { que son recién } \\
\text { graduados? }\end{array}$ & $\begin{array}{l}\text { Los jóvenes periodistas } \\
\text { salen de las aulas con } \\
\text { mente más fresca, se } \\
\text { entiende que con mayor } \\
\text { conocimiento } \\
\text { adaptación a la } \\
\text { tecnología }\end{array}$ & $\begin{array}{l}\text { Los egresados } \\
\text { tienen hambre por } \\
\text { la noticia, esas } \\
\text { ganas, esa vocación } \\
\text { fresca. }\end{array}$ & $\begin{array}{l}\text { Tienen deseos de } \\
\text { aprender y ponen } \\
\text { atención a lo que se } \\
\text { enseña en el } \\
\text { periódico que } \\
\text { difiere de lo que se } \\
\text { imparte en el aula } \\
\text { de clases. }\end{array}$ & $\begin{array}{lr}\text { La pasión por cubrir, } \\
\text { relatar, y publicar la } \\
\text { noticia. Los recién } \\
\text { egresados } & \text { están } \\
\text { ansiosos por } & \text { ganar } \\
\text { experiencia en } & \text { los } \\
\text { medios } & \text { de } \\
\text { comunicación } & \text { y } \\
\text { consolidar } & \text { su } \\
\text { posición en } & \text { este } \\
\text { mercado. Ofrecen } \\
\text { disponibilidad } & \text { de } \\
\text { tiempo y no } & \text { son } \\
\text { negativos a } & \text { las } \\
\text { disposiciones } & \text { o } \\
\text { asignaciones } & \\
\text { respecto } & \text { a } \\
\text { coberturas. } & \end{array}$ \\
\hline $\begin{array}{lr}\text { ¿Cuáles son las } \\
\text { debilidades } & \text { que } \\
\text { evidencian } & \text { los } \\
\text { pasantes } & \text { que } \\
\text { asisten a su } \\
\text { medio } & \text { de } \\
\text { comunicación o } \\
\text { los contratados } \\
\text { que son recién } \\
\text { graduados? }\end{array}$ & $\begin{array}{l}\text { No están acostumbrados } \\
\text { a leer a detalle las } \\
\text { noticias de actualidad, lo } \\
\text { que dificulta su } \\
\text { desempeño porque } \\
\text { carecen del contexto } \\
\text { informativo. Tienen } \\
\text { falencias en el uso del } \\
\text { lenguaje, y -a veces- } \\
\text { llegan a las salas de } \\
\text { redacción con ese } \\
\text { ímpetu de ser parte de } \\
\text { equipos periodísticos } \\
\text { especializados u obtener } \\
\text { cargos jerárquicos que } \\
\text { está bien; pero pierden el } \\
\text { norte cuando no } \\
\text { empiezan a sentar bases, } \\
\text { es decir, con la } \\
\text { reportería de calle. }\end{array}$ & $\begin{array}{lr}\text { Les } & \text { falta } \\
\text { experiencia; } & \text { a } \\
\text { veces se les enseña } \\
\text { más teoría y no } \\
\text { realizan talleres } \\
\text { prácticos que los } \\
\text { vinculen al mundo } \\
\text { laboral. } \\
\text { estudiantes } \\
\text { desconocen cómo } \\
\text { funciona } \\
\text { estructura de un } \\
\text { medio } & \text { la } \\
\text { comunicación. } & \end{array}$ & $\begin{array}{l}\text { Escasez de lectura, } \\
\text { desconocimiento } \\
\text { del contexto } \\
\text { cultural. } \\
\text { Falta de maestros } \\
\text { que aporten a su } \\
\text { profesión con el } \\
\text { rigor de haber } \\
\text { ejercido el oficio. }\end{array}$ & $\begin{array}{l}\text { La falta de } \\
\text { experiencia en la } \\
\text { cobertura } \\
\text { diferentes temas, no } \\
\text { manejan una buena } \\
\text { agenda de contactos } \\
\text { o fuentes, su } \\
\text { redacción dista de ser } \\
\text { buena. }\end{array}$ \\
\hline
\end{tabular}



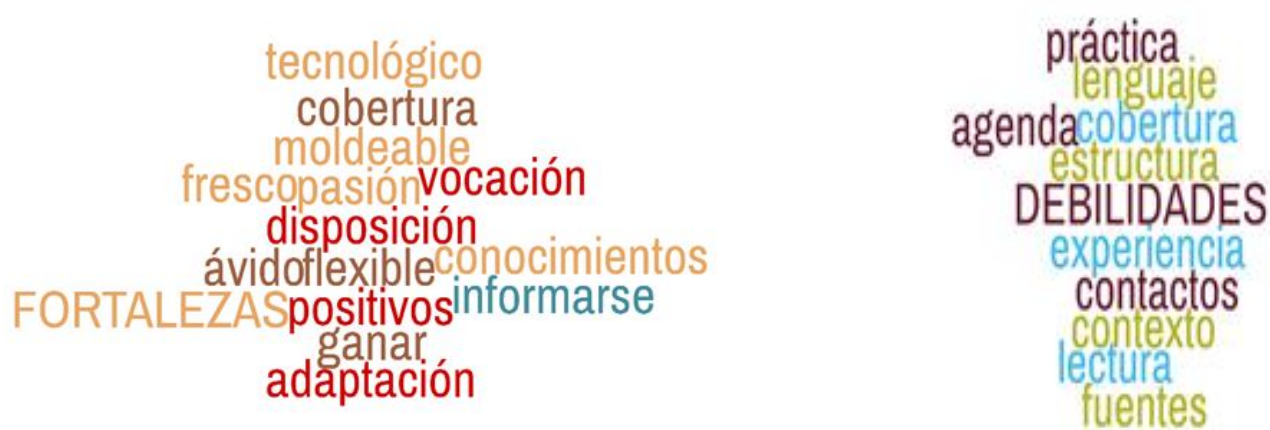

Figura 2. Fortalezas y debilidades que presentan los estudiantes que hacen prácticas en los medios de comunicación.

En las salas de redacción de los medios nacionales se requiere, además, desarrollar habilidades investigativas porque quien domina este aspecto marcará la pauta para desenvolverse en el resto de secciones. Marjorie Ortiz, es editora de sección de diario El Universo y sustenta la necesidad de conocer de leyes para entablar pertinentemente diálogos analíticos "Hay que desarrolladas las habilidades y herramientas en esta área. Por ejemplo, al analizar la evolución de casos de VIH SIDA en Ecuador desde 1984, se puede descubrir que la cifra ha aumentado en mujeres amas de casa; estos datos sirven para hacer un perfil de la enfermedad y evidenciar las formas de prevención de VIH SIDA en ese periodo y detectar si existen irregularidades o incumplimientos de la ley señalando a los responsables, a las autoridades que debieron velar por los derechos de los enfermos".

Contratar a un profesional multiplataforma que redacte la noticia y la transmita en tiempo real, que tenga conocimiento de herramientas multimedia y que puedan adaptarse a los formatos tecnológicos, que mantengan la pasión por la vocación y la entereza para no derrumbarse ante la complejidad de las tareas. Por ende, fortalecer el manejo del idioma y la redacción es la parte medular de un periodista quien deberá desarrollar habilidades tecnológicas que imperan en la era digital. Así también, es menester de la academia vincular al estudiante con la estructura de cada medio y la realidad de la profesión.

\section{La preparación académica en las universidades que atraviesan un proceso de categorización.}

Pero este dilema sobre el periodista tradicional y digital que se debate en las salas de redacción y en la web; es igual en la forma -no en el fondo- que tuvo hace más de 20 años cuando se debatía sobre la comunicación y el periodismo.

Algunas escuelas ofertaban Comunicación como profesión globalizada y que, incluso, contenía al Periodismo; pero, en las dos últimas décadas se ha disgregado estos términos. La tendencia ha sido la especialidad, dejando a la Comunicación como un campo amplio basado en la profundización de contenidos teóricos y al Periodismo como la práctica del oficio. En el ámbito académico Roveda Hoyos (2005) aclara que no existe tal división y que a pesar de que ambas carreras se ofertan de manera independiente, "hoy es entendida la formación de un comunicador como la profesión de los "grandes narradores de la contemporaneidad" y eso implica un excelente dominio de las formas de comunicación oral y escrita. 
El desarrollo de la oferta académica hizo que periodismo y comunicación se impartan de manera simultánea e, incluso, usando ambos términos como parte de un mismo accionar. Orientaciones curriculares diferentes que tienen el desafío de hilvanar armoniosamente técnicas periodísticas, bases teóricas y el estudio de la comunicación social. Según las encuestas realizadas en el estudio descriptivo: La voz de la academia: reflexiones sobre periodismo y comunicación, el 65,2\% de los docentes de Chile y Latinoamérica cree que la comunicación tiene un mayor campo disciplinario que el periodismo; no obstante, se complementan entre sí aunque se sentencia que "un periodista es comunicador social, pero que no todos los comunicadores son periodistas".

Las cifras muestran que el 8,7\% de los encuestados cree es lo mismo discutir de comunicación y periodismo; mientras que un $11,6 \%$ admite no tener claro el panorama y solo un $14,5 \%$ (Puerto Rico, Uruguay, Cuba y Argentina) dice que son cosas diferentes, enfatizando que periodismo es la técnica y la comunicación social la base teórica.

Un dato relevante es que al abordar sobre la producción de información por parte de la ciudadanía, el $50 \%$ se muestra a favor; esto también evidencia dos posturas: la primera, que el periodismo no puede renunciar a su estatus universitario; y, la segunda, la práctica de la carrera no se limita a la guía de periodistas titulados, sino que admite sujetos que sepan hacer bien su trabajo (Mellado, 2010).

Dicha sentencia "hombres titulados que sepan hacer su trabajo" coteja con el lineamiento de Pulitzer, quien acota que "hombres de periódicos que no saben nada de universidades u hombres de universidades que no saben nada de periódicos" es el panorama común que enfrentan las universidades que imparten esta disciplina. Él reflexiona sobre la complejidad de encontrar profesores competentes en la docencia; y aunque éste término denomina a alguien que conoce bien una técnica o disciplina; en la enseñanza del periodismo también exige experiencia.

El ejercicio profesional del periodismo atraviesa una crisis académica porque quien instruye, además de poseer títulos de tercer y cuarto nivel (licenciatura y maestría, respectivamente) habrá cursado las salas de los medios de comunicación; pues en ellos se forja la trayectoria, la verdadera valía de la profesión. En Ecuador, el Consejo de Educación Superior, determinó desde hace dos años que las instituciones universitarias establecieran el rediseño de las mallas académica y aunque ya están vigentes; sin profesores competentes, no hay plan curricular que garantice el éxito.

En la actualidad preocupa el rigor científico y la especialidad de la formación periodística y esto pone en riesgo el proceso de enseñanza y aprendizaje del periodismo. "Desempeñar un oficio y enseñarlo son dos cosas diferentes y aunque se requieran muchos conocimientos del primero para realizar lo segundo, no son suficientes (Vega \& Restrepo, 2015).

En la enseñanza de Periodismo debe primar "una combinación entre la más alta capacidad y reputación y la aptitud y la vocación por la enseñanza" y esto resulta lo más complicado, advierte el prestigioso editor y autor de su libro sobre periodismo. Añade también que los profesores de periodismo deberán ser redactores con experiencia; este es un desafío 
mayor pues quienes ejercen en medios no tienen maestría y tampoco el tiempo para realizarla debido al horario esquivo de trabajo resulta esquivo. Y quienes tienen título de cuarto nivel no han ejercido en medios, o no en impresos, cuyo rigor es exhaustivo.

Para lograr ser un buen periodista se necesita varios aspectos, uno de ellos es buscar información e ir a la fuente certera, contrastarla con investigación; esto y la redacción pulcra son las bases de un comunicador. El periodismo es presión; por eso, se necesita docentes que hayan vivido la experiencia de trabajar en medios y muestre la realidad de la profesión que les espera.

Existe una simbiosis: estudiantes que busquen la verdad y profesores que aporten con experiencia y aunque sería la fórmula perfecta para enseñar, detalla el prestigioso editor de Diario El País de España, Alex Grijelmo. Él explica que ser docente implica impartir teoría pero concentrarse en la investigación, en la buena composición; en buscar historias en la calle e insistir en los valores éticos de la profesión; solo de esta forma podrá conectar la formación con la realidad laboral.

"Se propone una enseñanza dialogada y reflexiva que abarque, en primer lugar, los conocimientos teóricos relativos a la naturaleza, objeto, elementos, medios y fines de la información periodística considerados en sí mismos, en su desarrollo histórico y en sus casos paradigmáticos... En el terreno de las aptitudes parece claro que los profesores de cada materia deben reunir todos los conocimientos previos que pretenden enseñar y poseer esas aptitudes que intentan forjar en los estudiantes, por la diáfana y sencilla razón de que nadie da lo que no tiene..." (Galdón, 1992).

Grijelmo también puntualiza sobre la vocación de los estudiantes. "Se busca estudiantes que busquen la verdad, que no se satisfactorio obtener un dato, sino que busque otro; que no se contente con una fuente, sino que salga a indagar dos; que no se satisfaga con una visión de la realidad, sino encuentra otras perspectivas". Advierte que para formar a las nuevas generaciones se requieren tres elementos: lectura, lectura y lectura. Sin embargo, la falta de interés por la misma se da por el mundo audiovisual que impera y que ahorra esfuerzos.

Según estudios del Centro Regional para el Fomento del Libro en América Latina y el Caribe (CERLALC) de la Unesco cada ecuatoriano lee medio libro por año. En ese mismo documento publicado en el 2012 informa que el país tiene un $43 \%$ de población lectora. Un porcentaje bajo en comparación a Argentina con un $85 \%$ y Chile $82 \%$. Quienes reciben a los pasantes de las universidades de los medios radial y escrito coinciden que hay graves falencias en la redacción. A esto se suman problemas para interpretar una noticia y a los tecnicismos de editar, usar un micrófono o temor a salir a una cobertura y hacer investigación. Docentes señalan que el principal problema es el poco interés en la lectura.

Facundo Landívar es editor general del diario El Clarín y El Mercurio de Argentina sentencia que: "sin universidad no hay periodismo" aunque el deterioro en la enseñanza es evidente. Esto se debe al porcentaje de profesores que no son periodistas sino solamente académicos que difícilmente vislumbran cuál es la realidad en una redacción, en la televisión o radio. Por eso, exhorta a que los buenos periodistas sean docentes pues es la única forma de transmitir una mejor enseñanza. 
La metodología idónea radica en el ejercicio de la profesión, no se puede enseñar si no está soportado por la experiencia, en la responsabilidad de enseñar con ejemplo porque los periodistas tienen una labora con la verdad, así reza la función social y nadie podría predicar aquello para lo que no se formó. La teoría es el complemento de la práctica y ambos elementos fortalecen los resultados: profesionales idóneos para los desafíos de la realidad.

\section{Conclusiones}

El profesional de hoy, además de dominar el lenguaje y redacción en diversos temas, debe centrar sus esfuerzos en especializarse en áreas determinadas, a fin de marcar una ventaja competitiva frente a otros profesionales afines. Las salas de redacción y departamentos de noticias demandan periodistas que no sólo trabajen por reportar, redactar la noticia del día, sino que sean capaces, además de cumplir aquello, transmitir en tiempo real la información, en consideración a la carrera de los medios por la inmediatez (Tabares, 2014). La especialización periodística es una oportunidad para ganar una vacante en los medios de comunicación, sean estos tradicionales o digitales.

El desafío de la nueva generación de periodistas es llegar a ser comunicadores multiplataforma. En la búsqueda de mejorar el perfil profesional precisa la adaptabilidad a las nuevas tecnologías y tendencias de comunicación y marketing; es menester el uso de las herramientas del periodismo de datos como una forma de diversificar la oferta de conocimientos y forjar un perfil profesional más atractivo. La academia juega un rol fundamental en la preparación de periodistas idóneos; por eso, es preciso sistematizar sus aportes y direccionamientos ante las demandas y exigencias del medio. La enseñanza del periodismo implica ser periodista. Esto no basta con la obtención de un título profesional sino del ejercicio del oficio porque la experiencia dotará al docente la facultad de hablar no solo de teoría sino de ampliar un contexto basado en el ejercicio de la realidad.

\section{Bibliografía}

Antón Bravo, A. (2013). El periodismo de datos y la web semántica. CIC. Cuadernos de Información $\quad y \quad$ Comunicación. $\quad$ Obtenido de http://www.redalyc.org/articulo.oa?id=93528051009

Ávila, A. M. (febrero de 2013). Periodismo de Datos: Historia y Momento Actual. Obtenido de http://manual.periodismodedatos.org/ana-maria-avila.php

Ávila, A. M. (s.f.). Manual de Periodismo de Datos Iberoamericano. Obtenido de http://manual.periodismodedatos.org/ana-maria-avila.php

Calvaresi, L., Valdivia, G., Foropón, D., \& Luna, M. (2010). Convergencia de redacciones: hacia la multiplataforma. Obtenido de Colegio Universitario de Periodismo, Argentina: https://periodismodigital.wikispaces.com/8.+Redacci\%C3\%B3n+convergente 
Cea, N. (2014). Presente y Futuro en el Periodismo Especializado. Ámbitos, revista andaluza de Comunicación. Núm. 25, 2-3.

Cordicom. (22 de enero de 2015). Las mujeres ganan espacio en los medios con profesionalismo y trabajo. Obtenido de http://www.cordicom.gob.ec/las-mujeres-ganan-espacio-en-losmedios-con-profesionalismo-y-trabajo/

El Ciudadano. (3 de enero de 2014). Ecuador recuerda el Día del Periodista con una comunicación participativa. Obtenido de http://www.elciudadano.gob.ec/ecuador-recuerda-el-dia-del periodista-con-una-comunicación-participativa/

Galdón, G. (1992). Cualidades y formación del periodista. Communication \& Society, Vol. 5 (1 y 2) $\quad 103-130$.

La-Rosa, L., \& Sandoval-Martín, T. (2016). La insuficiencia de la Ley de Transparencia para el ejercicio del Periodismo de datos en España. Revista Latina de Comunicación Social, (71),

1208-1229.

Obtenido

de file:///C:/Users/Julissa\%20Karina/Documents/JULISSA\%202017/Laica/Semestre\%20A $\% 20$

017.\%20Octubre/Syllabus\%20y\%20otros/Horas\%20de\%20escritura/La\%20insuficiencia $\% 2$

de\%20la\%20Ley\%20de\%20Transparencia\%20para\%20el\%20ejercicio\%20del\%20Period is $0 \% 20 \mathrm{de} \% 20 \mathrm{dat}$.ps.pd

Ley Orgánica de Comunicación. (Junio de 2013). Asamblea Nacional. Obtenido de http://www.asambleanacional.gob.ec/es/system/files/ley_organica_comunicacion.pdf

Mellado, C. (2010). La voz de la academia: reflexiones sobre periodismo y comunicación. Signo y Pensamiento, pp 274-287 volumen XXIX.

Nadjejda, V. (2009). Ayer, hoy y mañana de la información espacial: metamorfosis del periodismo especializado en la era espacial. Obtenido de https://ebookcentral.proquest.com

Pulitzer, J. (2015). Joseph Pulitzer sobre periodismo. Gallo Nero.

Punín, M., \& Martínez, A. (4 de enero de 2013). La profesionalización periodística en Ecuador, la experiencia en las calles o el conocimiento de las aulas. Obtenido de https://revistas.ucm.es/index.php/ESMP/article/viewFile/42535/40444

Scolari, C., Micó, J., Navarro, H., \& Pardo, H. (2008). El periodista polivalente. Transformaciones en el perfil del periodista a partir de la digitalización de los medios audiovisuales catalanes. Zer, Volumen 13. Núm. 25 pp. 37-60.

Tabares, L. (2014). Discusiones sobre la enseñanza de periodismo. Revista Comunicación, No. 31. Enero-diciembre p. 67 - 74. 
Valle, A. (16 de julio de 2014). Periodismo Especializado. La Razón, pág. Tribuna. Obtenido de https://www.larazon.es/opinion/tribuna/periodismo-especializado-EB6928868

Vega, M., \& Restrepo, M. (2015). Periodismo. Enseñanza y Aprendizaje. Revista Luciérnaga.

Facultad de Comunicación Audiovisual. Universidad Autónoma de San Luis Potosí., Año 7, Edición 14. págs.: 78-99. Medellín-Colombia.

Vicente, N. (2009). Ayer, hoy y mañana de la información espacial: metamorfosis del periodismo especializado en la era espacial. Obtenido de https://ebookcentral.proquest.com 